\title{
Erratum to: Laparoscopic gastrectomy for gastric cancer in the elderly patients
}

\author{
Muneharu Fujisaki $^{1} \cdot$ Toshihiko Shinohara $^{1} \cdot$ Nobuyoshi Hanyu $^{1} \cdot$ Susumu Kawano $^{1}$. \\ Yujiro Tanaka $^{1}$ Atsushi Watanabe ${ }^{1} \cdot$ Katsuhiko Yanaga $^{2}$
}

Published online: 14 November 2016

(c) Springer Science+Business Media New York 2016

\section{Erratum to: Surg Endosc (2016) 30:1380-1387 \\ DOI 10.1007/s00464-015-4340-5}

Tables 1, 4, and 5 had the following corrections implemented:

“3 7 (10.0) 6 (4.9)" should be " $\geq 37$ (10.0) 6 (4.9)" (on p. 1383, line 11 in Table 1).

“3 8 (4.6) 5 (27.8)" should be " $\geq 38$ (4.6) 5 (27.8)" (on p. 1385, line 14 in Table 4).

"D2 3 (36.0) 7 (38.9)" should be "D2 63 (36.0) 7 (38.9)" (on p. 1385, line 29 in Table 4).

"3 $0.03414 .441 .22-170.56$ " should be " $\geq 3 \quad 0.034$ 14.44 1.22-170.56" (on p. 1386, line 6 in Table 5).

The corrected tables are displayed below:

The online version of the original article can be found under doi: 10.1007/s00464-015-4340-5.

Toshihiko Shinohara shinohara@jikei.ac.jp

1 Department of Surgery, Machida Municipal Hospital, 2-15-41 Asahicho, Machida, Tokyo 194-0023, Japan

2 Department of Surgery, The Jikei University School of Medicine, Tokyo, Japan 
Table 1 Patient characteristics

\begin{tabular}{|c|c|c|c|}
\hline & Elderly $(n=70)$ & Non-elderly $(\mathrm{n}=123)$ & $P$ value \\
\hline Age $(\text { years })^{\mathrm{a}}$ & $80.1(4.1)$ & $64.8(7.5)$ & $<0.001$ \\
\hline $\operatorname{Sex} n(\%)$ & & & 0.849 \\
\hline Male & $44(62.9)$ & $79(64.2)$ & \\
\hline Female & $26(37.1)$ & $44(35.8)$ & \\
\hline BMI $\left(\mathrm{kg} / \mathrm{m}^{2}\right)^{\mathrm{a}}$ & $22.3(2.6)$ & $22.6(3.2)$ & 0.523 \\
\hline CCI $n(\%)$ & & & 0.228 \\
\hline 0 & 34 (48.6) & $68(55.3)$ & \\
\hline 1 & $18(25.7)$ & $38(30.9)$ & \\
\hline 2 & $11(15.7)$ & $11(8.9)$ & \\
\hline$\geq 3$ & $7(10.0)$ & $6(4.9)$ & \\
\hline ASA score $n(\%)$ & & & 0.018 \\
\hline 1,2 & $57(81.4)$ & $114(92.7)$ & \\
\hline 3 & 13 (18.6) & $9(7.3)$ & \\
\hline Tumor size $(\mathrm{mm})^{\mathrm{a}}$ & $35.6(25.9)$ & $37.8(31.6)$ & 0.605 \\
\hline Histological type $n(\%)$ & & & 0.220 \\
\hline Differentiated & $40(57.1)$ & $59(48.0)$ & \\
\hline Undifferentiated & $30(42.9)$ & $64(52.0)$ & \\
\hline TNM stage $n(\%)^{\mathrm{b}}$ & & & 0.518 \\
\hline I & $49(70.0)$ & $96(78.0)$ & \\
\hline II & $11(15.7)$ & $15(12.2)$ & \\
\hline III & $8(11.4)$ & $11(8.9)$ & \\
\hline IV & $2(2.9)$ & $1(0.8)$ & \\
\hline
\end{tabular}

$B M I$ body mass index, $C C I$ Carlson Comorbidity Index, ASA American Society of Anesthesiologists

${ }^{\text {a }}$ Values are shown as means (standard deviations)

b According to the UICC staging [11] 
Table 4 Univariate analysis of risk factors for postoperative complications

\begin{tabular}{|c|c|c|c|}
\hline & \multicolumn{2}{|l|}{ Complications } & \multirow[t]{2}{*}{$P$ value } \\
\hline & No $(n=175)$ & Yes $(n=18)$ & \\
\hline Age (years) $n(\%)$ & & & 0.449 \\
\hline$<75$ & $113(64.6)$ & $10(55.6)$ & \\
\hline$\geq 75$ & $62(35.4)$ & $8(44.4)$ & \\
\hline $\operatorname{Sex} n(\%)$ & & & 0.431 \\
\hline Male & $110(62.9)$ & $13(72.2)$ & \\
\hline Female & $65(37.1)$ & $5(27.8)$ & \\
\hline Mean BMI $\left(\mathrm{kg} / \mathrm{m}^{2}\right)^{\mathrm{a}}$ & $22.4(2.9)$ & $23.2(3.5)$ & 0.407 \\
\hline CCI $n(\%)$ & & & 0.002 \\
\hline 0 & $95(54.3)$ & $7(38.9)$ & \\
\hline 1 & $51(29.1)$ & $5(27.8)$ & \\
\hline 2 & $21(12.0)$ & $1(5.6)$ & \\
\hline$\geq 3$ & $8(4.6)$ & $5(27.8)$ & \\
\hline ASA score $n(\%)$ & & & 0.008 \\
\hline 1,2 & $159(90.9)$ & $12(66.7)$ & \\
\hline 3 & $16(9.1)$ & $6(33.3)$ & \\
\hline Operative time (min) & & & 0.004 \\
\hline$<330$ & $118(67.4)$ & $6(33.3)$ & \\
\hline$\geq 330$ & $57(32.6)$ & $12(66.7)$ & \\
\hline Intraoperative blood loss (ml) & & & 0.006 \\
\hline$<50$ & $107(61.1)$ & $5(27.8)$ & \\
\hline$\geq 50$ & $68(38.9)$ & $13(72.2)$ & \\
\hline Operative method & & & 0.120 \\
\hline Distal gastrectomy & $148(84.6)$ & $12(66.7)$ & \\
\hline Total gastrectomy & $27(15.4)$ & $6(33.3)$ & \\
\hline Lymph node dissection $^{\mathrm{b}}$ & & & 1.000 \\
\hline D1 or D1+ & $112(64.0)$ & $11(61.1)$ & \\
\hline D2 & $63(36.0)$ & $7(38.9)$ & \\
\hline TNM stage, $n(\%)^{\mathrm{c}}$ & & & 0.662 \\
\hline I & $133(76.0)$ & $12(66.7)$ & \\
\hline II & $23(13.1)$ & $3(16.7)$ & \\
\hline III & $16(9.1)$ & $3(16.7)$ & \\
\hline IV & $3(1.7)$ & $0(0.0)$ & \\
\hline Year of operation $(\%)$ & & & 0.485 \\
\hline Early period (January 2007-June 2010) & $54(30.9)$ & 7 (38.9) & \\
\hline Late period (July 2010-September 2014) & $121(69.1)$ & $11(61.1)$ & \\
\hline
\end{tabular}

$B M I$ body mass index, $C C I$ Carlson Comorbidity Index, ASA American Society of Anesthesiologists

${ }^{a}$ Values are shown as means (standard deviations)

b According to the treatment guidelines issued by the Japanese Cancer Association [6]

c According to the UICC staging [11] 
Table 5 Multivariate analysis of risk factors for postoperative complications

\begin{tabular}{llll}
\hline \multicolumn{1}{c}{$P$ value } & Odds ratio & $95 \%$ CI \\
\hline CCI & & & \\
0 & - & 1 & - \\
1 & 0.093 & 4.17 & $0.79-22.09$ \\
2 & 0.219 & 2.89 & $0.53-15.64$ \\
$\geq 3$ & 0.034 & 14.44 & $1.22-170.56$ \\
ASA score & & & \\
1,2 & - & 1 & - \\
3 & 0.019 & 6.42 & $1.37-30.22$ \\
Operative time (min) & & & \\
$\quad<330$ & - & 1 & - \\
$\geq 330$ & 0.149 & 2.32 & $0.74-7.31$ \\
Intraoperative blood loss (ml) & & & \\
$<50$ & - & 1 & - \\
$\geq 50$ & 0.016 & 5.23 & $1.37-19.94$ \\
\hline CCI Carlson Comorbidity & Index, ASA & American & Society of \\
Anesthesiologists & & &
\end{tabular}

